\title{
Cinnamophilin Inhibits Neutrophilic Respiratory Burst and Protects Against Ischemia-Reperfusion Brain Damage
}

Yu-Wen Lin', Shih-Huang Tai', Chih-Hao Tien', Sheng-Yang Huang', Che-Chao Chang', Tian-Shung Wu², Wei-Sheng Juan', Hung-Yi Chen ${ }^{3}$ and E-Jian Lee ${ }^{1 *}$

${ }^{1}$ Neurophysiology Laboratory, Neurosurgical Service, Department of Surgery, National Cheng Kung University Hospital, College of Medicine, National Cheng Kung University, Tainan, Taiwan

${ }^{2}$ Department of Chemistry, National Cheng Kung University, Tainan, Taiwan

${ }^{3}$ China Medical University, Institute of Pharmacy, Taichung, Taiwan

\begin{abstract}
We have shown that administration of Cinnamophilin (CINN) effectively reduced oxidative damage, DNA lipid peroxidation, neutrophil infiltration, and ischemic brain damage by inhibiting oxidative stress and the resulting inflammation in experimental stroke. In this study the potential CINN to ameliorate neutrophilic respiratory burst and reduce neutrophil infiltration was investigated. Neutrophils pretreated or co-treated with CINN, were stimulated by phorbol 12-myristate 13-acetate (PMA) and the levels of superoxide radical $\left(\mathrm{O}_{2}-\right.$.) and hydrogen peroxide $\left(\mathrm{H}_{2} \mathrm{O}_{2}\right)$ produced were determined by dihydroethidium (DHE) and dihydrorhodamine-123 (DHR) fluorescence assays, respectively, while myeloperoxidase activity (MPO) was measured by the guaiacol method. Our results showed that both pretreatment and co-treatment with CINN significantly inhibited $\mathrm{H}_{2} \mathrm{O}_{2}$ production in PMA-stimulated neutrophils. Additionally, cotreatment, but not pretreatment, with $\mathrm{CINN}$ effectively inhibited $\mathrm{O}_{2}$-. production in the PMA-stimulated neutrophils. Both treatments did not effectively reduce the MPO activity in neutrophil. Finally, animals treated with CINN at reperfusion brain insults significantly reduced brain infarction and neutrophil infiltration, as well as improved neurobehavioral outcome following cerebral ischemic reperfusion. These results support pluripotent neuroprotection actions offered by CINN against cerebral ischemia-reperfusion.
\end{abstract}

Keywords: Cinnamophilin; Neutrophilic respiratory burst; Cerebral ischemia-reperfusion

\section{Introduction}

Up to $40 \%$ of stroke patients did not recover their independence [1], which makes stroke a major public issue in the world. There are limited therapeutic treatments, available in clinic, because administration of tissue plasminogen activator (tPA) within 3 hours of symptom onset only benefits a small proportion of stroke patients [2-4], and also increases a risk of hemorrhagic transformation which may potentiate ischemic neuronal damage [5-9]. Thus, there is an urgent need for safe and effective neuroprotective agents that can beneficial for the majority of stroke patients.

It is well known that neutrophils play an important role in the pathogenesis of ischemia reperfusion injury by releasing a variety of oxygen radicals including $\mathrm{O}_{2}^{-.}, \mathrm{H}_{2} \mathrm{O}_{2}$, and $\mathrm{OH}-$. [10,11].

Following cerebral ischemia-reperfusion, neutrophils produce a variety of reactive oxygen species (ROS) when they are activated during inflammatory responses [12], a process which is known as the neutrophilic respiratory burst. NADPH oxidase which is located in neutrophils catalyzes the generation of $\mathrm{O}_{2}-$. from oxygen and $\mathrm{NADPH}$ [13-15] while $\mathrm{O}_{2}-$. is converted to $\mathrm{H}_{2} \mathrm{O}_{2}$ by superoxide dismutase (SOD). Neutrophils utilize myeloperoxidase to further combine $\mathrm{H}_{2} \mathrm{O}_{2}$ with chlorides to produce hypochlorite. Therefore, neutrophilic respiratory burst results in the release of $\mathrm{O}_{2}-$. and $\mathrm{H}_{2} \mathrm{O}_{2}$, which play an important mechanism of cerebral ischemia-reperfusion injury. It has been shown that neutrophil depletion or inhibition of neutrophil function exerted significant protective effects in ischemia reperfusioninduced brain injury [16-19]. One strategy to protect the brain against ischemic-reperfusion injury is to improve the endogenous antioxidant defense of the tissues at risk, to decrease oxidative damage by scavenging toxic free radicals [20-22] and to inhibit neutrophilic respiratory burst in activated neutrophils [23]. Cinnamophilin, (CINN;
[8R, 8S]-4, 4-dihydroxy-3, 3-dimethoxy-7-oxo-8, 8-neolignan), is a highly lipophilic compound isolated from Cinnamomum philippinense, which can readily penetrate the blood-brain barrier with a slow decay in the brain $[24,25]$. We have previously demonstrated that CINN not only reduced brain infarction and oxidative stress, but also improved behavioral outcome in a mouse model of transient middle cerebral artery (MCA) occlusion [26,27]. More recently, we have demonstrated that CINN exhibited anti-inflammatory actions against ischemic stroke [26]. In the present study, we further examined whether administration of CINN would reduce brain inflammation by inhibiting neutrophilic respiratory burst.

\section{Materials and Methods}

\section{Animals}

C57BL/B6 mice, weighted 19-22 g, and Sprague-Dawley rats, weighed 250-280 g, were supplied by the University Laboratory Animal Center. All procedures performed were approved by the Subcommittee on Research Animal Care of the University Medical Center, and the standards meet the guidelines of the National Institutes of Health.

*Corresponding author: E-Jian Lee, Department of Surgery, National Cheng Kung University Medical Hospital, 138 Sheng-Li Road, Tainan, Taiwan, Tel: +8866-235-3535 ext.5186; Fax: +886-6-276-6676; E-mail: ejian@mail.ncku.edu.tw

Received October 19, 2013; Accepted November 18, 2013; Published November 21, 2013

Citation: Lin YW, Tai SH, Tien CH, Huang SY, Chang CC, et al. (2013) Cinnamophilin Inhibits Neutrophilic Respiratory Burst and Protects Against Ischemia-Reperfusion Brain Damage. Pharmaceut Anal Acta 4: 272. doi: 10.4172/2153-2435.1000272

Copyright: $\odot 2013$ Lin YW, et al. This is an open-access article distributed under the terms of the Creative Commons Attribution License, which permits unrestricted use, distribution, and reproduction in any medium, provided the original author and source are credited. 
Citation: Lin YW, Tai SH, Tien CH, Huang SY, Chang CC, et al. (2013) Cinnamophilin Inhibits Neutrophilic Respiratory Burst and Protects Against Ischemia-Reperfusion Brain Damage. Pharmaceut Anal Acta 4: 272. doi: 10.4172/2153-2435.1000272

\section{Chemicals and reagents}

All chemicals were purchased from Sigma-Aldrich Co. (St Louis, MO) unless otherwise indicated. CINN was isolated from the root of Cinnamomum philippinense by the methods previously described [28] and was dissolved in $0.1 \%$ dimethylsulfoxide (DMSO, Sigma-Aldrich Co.) for in vitro studies

\section{Transient middle cerebral artery occlusion model}

Adult Sprague-Dawley rats were anesthetized with $1 \%$ halothane in $70 \% \mathrm{~N}_{2} \mathrm{O} / 30 \% \mathrm{O}_{2}$. Animals were subjected to intra-arterial filament occlusion of the right proximal middle cerebral artery (MCA) for 90 minutes by the methods previously described [29]. During surgery, animals' rectal temperatures were maintained at $37.0 \pm 1.0^{\circ} \mathrm{C}$ using a thermostatically controlled heating blanket (Harvard Apparatus, South Natick, MA, USA) and heating lamp. Effective MCA occlusion was confirmed by the measurements of a Laser-Doppler flowmetry (LaserFlo BMP2, Vasamedics, St. Paul, MN, USA). Sham-operated rat received similar surgical procedures excluding vascular opening and filament insertion.

\section{Drug administration}

Animals were randomly assigned to treatment protocol. The investigators were blinded to the treatment paradigm. CINN was dissolved in $45 \%$ aqueous hydroxypropyl- $\beta$-cyclodextrin (HPCD). An optimal dosage of CINN at $80 \mathrm{mg} / \mathrm{kg}$ was chosen based on its neuroprotective dose-response studies in a rodent model of stroke [27]. Animals were administered intravenously with CINN $(80 \mathrm{mg} / \mathrm{kg})$ or the same volume of HPCD at 2 hours after the onset of MCA occlusion.

\section{Neurobehavioral testing}

Body weight measurements and neurologic evaluations were assessed at 72 hours after the ischemia-reperfusion insult by one observer unaware of treatment protocol. Two neurologic grading systems were used: (i) a sensorimotor grading scale modified from previously published methods [30] with five categories (0-4) for forward and sideways visual placing tests of the affected forelimb and five categories (0-4) for motor outcome and (ii) a grading scale of 0-28 developed by Clark et al. [31].

\section{Animal sacrifice and quantification of ischemic damage}

Following 72 hours of survival, animal wassacrificed underanesthesia by transcardiac perfusion accomplished with $4 \%$ paraformaldehyde in $0.1 \mathrm{M}$ phosphate-buffered saline. Brains were removed, stored in the same fixative for 24 hours, and sequentially immersed in 15 and 30\% sucrose at $4^{\circ} \mathrm{C}$ for 48 hours. The brains were then embedded in Optimal Cutting Temperature compound (OCT, Miles Inc., Elkhart, IN, USA) and frozen in liquid nitrogen. The brains were sectioned coronally on a cryostat (HM-500O, Microm International GmbH, Walldorf, Germany). Serial sections of $40 \mu \mathrm{m}$ at eight preselected coronal levels, with 1-mm intervals from the stereotaxic coordinates of the Bregma AP +2.22 to $-4.78 \mathrm{~mm}$, were mounted on poly-l-lysine-coated slides and dried at $37^{\circ} \mathrm{C}$ overnight. Brain infarction was determined by staining preselected brain slices with hematoxylin and eosin stain (HE). Under light microscopy, the areas of neuronal perikarya displaying typical morphological features of ischemic damage were delineated. Infarction volume was measured using a computerized image analyzer (MCID Elite, Imaging Research Inc., Ontario, Canada) and was expressed as a percentage of the contralateral hemisphere volume [29].

\section{Detection of free radicals using dihydroethidium in situ}

Brain sections were incubated with dihydroethidium (DHE) (stock solution, $1 \mathrm{mM}$ in dimethyl sulfoxide, diluted to $5 \mu \mathrm{m}$ with PBS, Molecular Probes, Eugene, OR, USA) in a humidified chamber, at $37^{\circ} \mathrm{C}$ for 30 minutes. The sections were rinsed in PBS for 15 minutes and incubated with DAPI in PBS for 15 seconds in a dark chamber, and then were rinsed in PBS and mounted with Aquamount. Fluorescence was assessed at excitation $365 \mathrm{~nm}$ and emission $>420 \mathrm{~nm}$ for DAPI and was at excitation $510-550 \mathrm{~nm}$ and emission $>580 \mathrm{~nm}$ for ethidium detection. The intensity of the oxidized HEt on the ischemic brain was observed and compared between the CINN-treated and vehicle-treated animals.

\section{Immunohistochemistry for myeloperoxidase}

Coronal brain sections were cut into $40 \mu \mathrm{m}$. A set of brain sections was processed for immunohistochemistry with polyclonal rabbit antimyeloperoxidase antibody (1:200, Thermo Fisher Scientific, Fremont, CA, USA) at $4^{\circ} \mathrm{C}$ overnight. After washes with PBS for 5 minutes three times, the bound antibody was visualized by labeling with goat antirabbit immunoglobulin G-fluorescein isothiocyanate (FITC) conjugate (Molecular Probes) as a fluorescent probe. Fluorescence was assessed at excitation $488 \mathrm{~nm}$ and emission $520 \mathrm{~nm}$ for FITC detection.

\section{Neutrophils isolation}

Mice wereintraperitoneallyinjected with $5 \mathrm{mg} / \mathrm{kglipopolysaccharide}$ (LPS) to induce neutrophils in blood. Cardiac blood collected 24 hours later was incubated with ACK buffer $(0.15 \mathrm{M} \mathrm{NH} 4 \mathrm{Cl}, 1 \mathrm{mM} \mathrm{KHCO}$, and $0.1 \mathrm{mM}$ EDTA) for 6 minutes, followed by centrifugation at $800 \mathrm{~g}$ to remove red blood cells. Cells were then washed with Hank's balanced salt solution (HBSS, GIBCO, Grand Island, NY, USA) containing $0.05 \%$ bovine serum albumin (BSA).

\section{The cytotoxic effect of CINN on neutrophils}

For cytotoxicity investigations, neutrophils were pre-incubated with a fixed concentration of CINN (1-300 $\mu \mathrm{m})$, phorbol 12-myristate 13 -acetate (PMA; $20 \mathrm{ng} / \mathrm{ml})$ or vehicle $(0.1 \% \mathrm{DMSO})$ for 30 minutes. Cytotoxicity was determined by the propidium iodine (PI; $50 \mu \mathrm{g} / \mathrm{mL}$ ) staining at 24 hours after treatment.

\section{Respiratory burst assay in neutrophils}

PMA (20 ng/ml) was added for 20 minutes to induce the production of $\mathrm{H}_{2} \mathrm{O}_{2}$ and $\mathrm{O}_{2}-$. Neutrophils were either pretreated or cotreated with CINN at different doses (1-100 $\mu \mathrm{M})$ for 15 minutes followed by staining with dihydrorhodamine-123 (DHR-123;FL1) to label $\mathrm{H}_{2} \mathrm{O}_{2}$ or with dihydroethidium (DHE; FL2) to label $\mathrm{O}_{2}$-. at a final concentration of 10 $\mu \mathrm{M}$ for 5 minutes. Neutrophils were gated based on the FSC/SSC scatter on a FACSCalibur cytometer (BD Biosciences, San Jose, CA, USA) and analyzed using the Cell Quest software (BD Biosciences, San Jose, CA, USA).

\section{Statistical analysis}

Data were presented as the mean \pm standard deviation of the mean (S. D.). The distribution of the date was verified by Levene tests before post hoc comparisons. Cytotoxic effects of CINN on the viability of neutrophil were analyzed by one-way analysis of variance (one-way ANOVA) with LSD protected least significant difference post hoc comparison. The data of respiratory burst of neutrophils were analyzed by Kruskal-Wallis/Mann-Whitney U tests because they were not found to follow normal distribution. The other data with normal/ 
Citation: Lin YW, Tai SH, Tien CH, Huang SY, Chang CC, et al. (2013) Cinnamophilin Inhibits Neutrophilic Respiratory Burst and Protects Against Ischemia-Reperfusion Brain Damage. Pharmaceut Anal Acta 4: 272. doi: 10.4172/2153-2435.1000272

approximately normal distribution were analyzed by using unpaired Student's $t$ test. $\mathrm{P}<0.05$ was selected for statistical significance.

\section{Results}

CINN reduced brain infarction following MCA occlusion at 3 days

Animal treated either with CINN or vehicle did not have altered local cortical blood perfusion or core temperature during a course of surgery (data not shown). Relative to controls, animals treated with CINN had significant reduced brain infarction and hemispheric edema. Treatment with CINN resulted in $22.9 \%$ reduction in infarction volumes $(P<0.05$; Figure $1 \mathrm{~A}$ and $1 \mathrm{~B})$. However, CINN neither significantly reduced brain swelling nor effectively affected individual cortical and striatal infarcts (Figure $1 \mathrm{~B}$ and $\mathrm{C} ; P>.05$, respectively).

CINN inhibited superoxide production and neutrophil infiltration in the brain of animals subjected to transient MCA occlusion

Intracellular production of $\mathrm{O}_{2}-$. was measured by the staining of DHE. CINN administration significantly decreased DHE signals in the ischemic brain sections (Figure $2, \mathrm{P}<0.05$ ). Thus, CINN could attenuate the in situ accumulation of $\mathrm{O}_{2}-$. in the ischemic brain. In vehicle-treated controls, the ischemic reperfusion insult induced dramatically increased numbers of the MPO-expressing cells in the ischemic territory. CINNtreatment animals, however, significantly reduced the number of MPOimmunopositive cells, compared with controls (Figure 3, $\mathrm{P}<0.05$ ).

\section{Effect of CINN on cell viability of neutrophils}

The cell viability was determined by the propidium iodine assay.

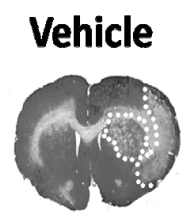

\section{CINN}

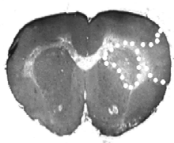

C
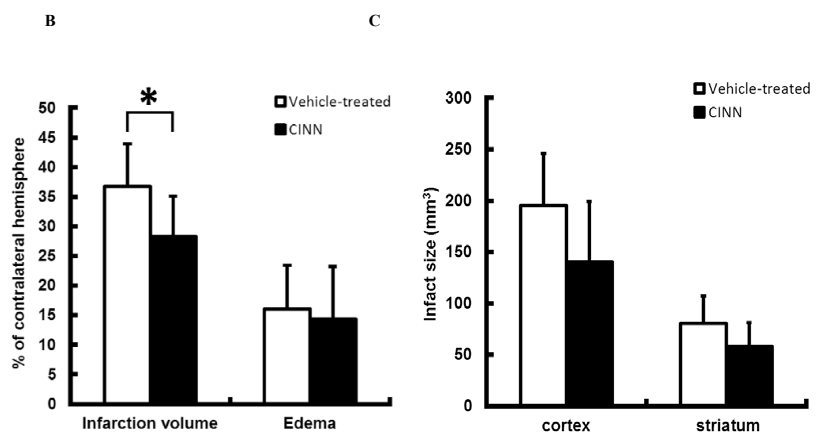

Figure 1: Cinnamophilin reduced brain infarction and swelling at 3 days after transient focal cerebral ischemia. Rats received either CINN $(80 \mathrm{mg} /$ $\mathrm{kg}$ ) or vehicle (the same volume of HPCD) at 2 hours after MCA occlusion. (A) Representative hematoxylin and eosin stained sections from the vehicleinjected and the CINN-treated animals. (B) CINN significantly decreased brain infarction volumes and swelling and reduced $(C)$ individual cortical and striatal lesion sizes. Data are presented as mean \pm standard deviation (S. D.), and $n=6$ per group. ${ }^{*} P<0.05$ compared with the vehicle-injected controls by using the unpaired Student's $t$ test.
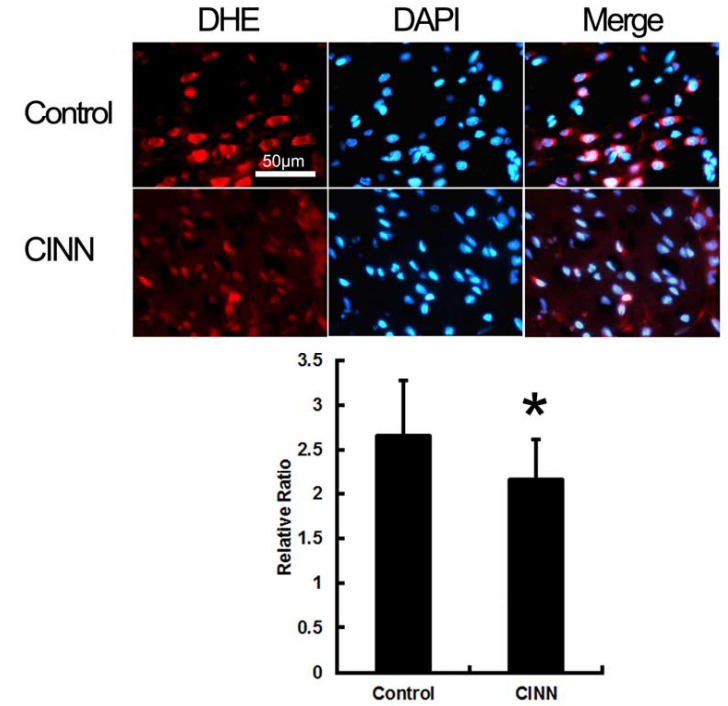

Figure 2: Cinnamophilin decreased superoxide radical production in the ischemic brain. In situ detection of intracellular superoxide radical production was measured by dihydroethidium (DHE) in the ischemic brain. The brain sections were double stained with DHE (red) and DAPI staining (blue). The CINN-treated rats had a significant decrease in the intensity of DHE signals, compared to the the vehicle-injected controls. Data are presented as mean \pm standard deviation (S. D.), and $\mathrm{n}=6$ per group. ${ }^{*} P<0.05$ compared with vehicletreated controls by using the unpaired Student's $t$ test.
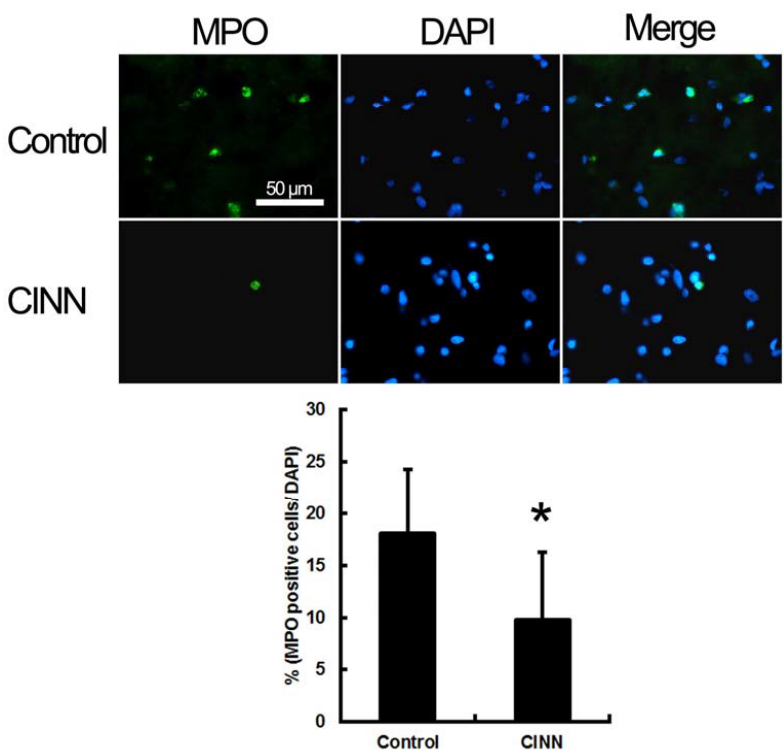

Figure 3: Cinnamophilin inhibited neutrophil infiltration in the ischemic brain The brain sections were double stained with myeloperoxidase (MPO) and DAPI staining. The CINN-treated rats had marked decreases in the number of the MPO-expressing cells. Data are presented as mean \pm standard deviation (S. D.), and $n=6$ per group. ${ }^{*} P<0.05$ compared with the vehicle-injected controls by using the unpaired Student's $t$ test.

No significant cytoxicity to neutrophils was found with CINN at the concentrations of 1 to $100 \mu \mathrm{M}$ (Figure 4). CINN (300 $\mu \mathrm{M})$, however, exhibited increased cytoxicity to neutrophils. 


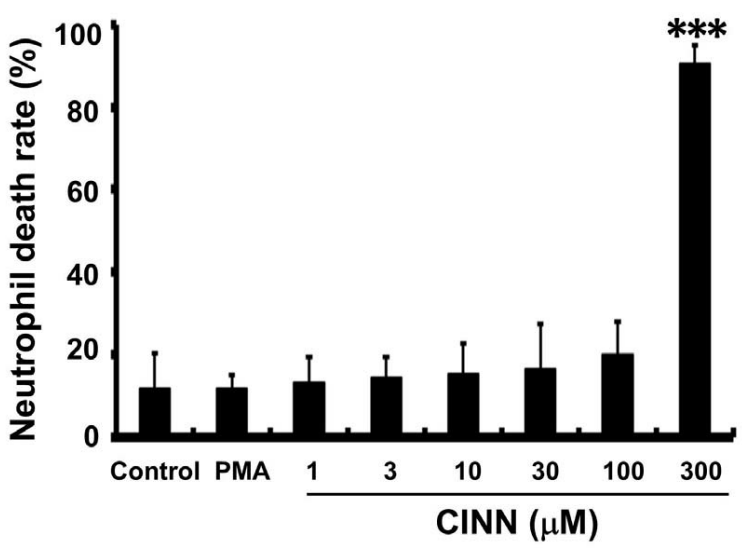

Figure 4: Cytotoxic effect of cinnamophilin on the viability of neutrophil. The cells were treated with PMA $(20 \mathrm{ng} / \mathrm{ml})$ or CINN $(1$ to $300 \mu \mathrm{M})$ for 24 hours and the viability of neutrophils were determined by propidium iodine stained. Data are expressed by the mean \pm standard deviation (S. D.), and $n=3$ per group. ${ }^{* * *} P<0.001$ compared with vehicle-treated controls by using one-way analysis of variance (one-way ANOVA) with LSD protected least significant difference post hoc comparison.

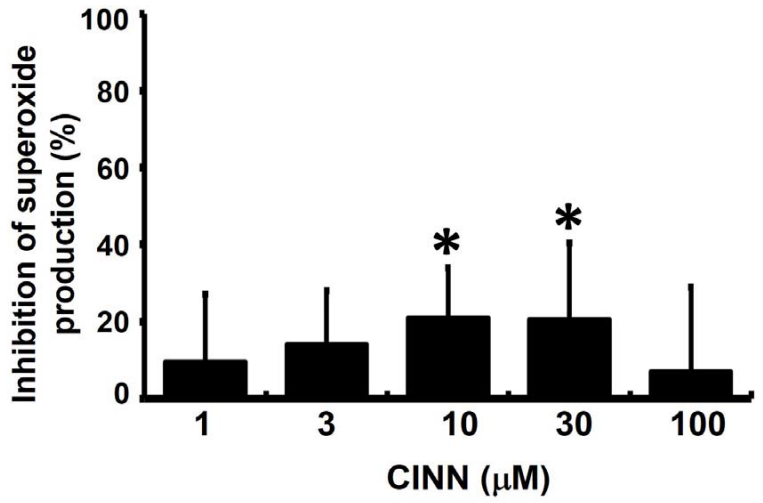

Figure 5: Inhibitory effects of cinnamophilin on superoxide radical production in the PMA-treated neutrophils. The cells were cotreated with CINN (1 to 100 $\mu \mathrm{M})$ and stimulated with PMA for 15 minutes followed by staining with DHE. The superoxide radical production of cells was determined by flow cytometric assay. Data are expressed by the mean \pm standard deviation (S. D.), and $n=6$ per group. ${ }^{*} P<0.05$ compared with controls by using the Kruskal-Wallis/MannWhitney $U$ tests.

Cotreatment with CINN inhibited superoxide radical production in the PMA-stimulated neutrophils

Pretreatment with CINN at 1-300 $\mu \mathrm{M}$ had no effect on $\mathrm{O}_{2}$ production of neutrophil after the PMA stimulation (data not shown). Cotreatment with CINN at 10-30 $\mu \mathrm{M}$ effectively reduced the $\mathrm{O}_{2}$-. production by $21 \%$ in the PMA-stimulated neutrophils (Figure 5 , $P<0.05)$.

\section{Pretreatment or cotreatment with CINN inhibited hydrogen peroxide production in neutrophils}

Pretreatment with CINN at 3-100 $\mu \mathrm{M}$ dose-dependently inhibited $\mathrm{H}_{2} \mathrm{O}_{2}$ production by $18-77 \%$ in the PMA-stimulated neutrophils (Figure 6A, $P<0.05$ ). Cotreatment with CINN at 3-100 $\mu \mathrm{M}$ also dose-dependently inhibited $\mathrm{H}_{2} \mathrm{O}_{2}$ production by $5-88 \%$ in the PMA-stimulated neutrophils (Figure $6 \mathrm{~B}, \mathrm{P}<0.05$ ). The $\mathrm{IC}_{50}$ for $\mathrm{H}_{2} \mathrm{O}_{2}$ production was $25.4 \mu \mathrm{M}$ and $13.9 \mu \mathrm{M}$ in the pretreatment and cotreatment CINN protocols, respectively. We further investigated the effect of CINN on the MPO activity in the PMA-stimulated neutrophils, and have observed that CINN had no effect on the MPO activity in the PMA-stimulated neutrophils (data not shown).

\section{Discussion}

Our results demonstrated that $\mathrm{CINN}$ could directly inhibit $\mathrm{O}_{2}-$. and $\mathrm{H}_{2} \mathrm{O}_{2}$ produced by the PMA-induced respiratory burst in neutrophils. Although CINN did not directly suppress the MPO activity of neutrophils, the agent effectively reduced the MPO activity induced by cerebral ischemia-reperfusion injury observed in vivo (Figure 3). Thus, CINN could protect the brain from ischemia-reperfusioninduced injury by inhibiting the respiratory burst actions in activated neutrophils.

We have demonstrated that CINN ( $80 \mathrm{mg} / \mathrm{kg}$ ) effectively reduced brain damage induced by cerebral ischemic reperfusion insults in rats [27]. Additionally, the agent effectively improved the numbers of the surviving neurons in the penumbral parietal cortex and caudoputamen [27]. Our previous works have also demonstrated that CINN effectively scavenged free radicals and improved the endogenous antioxidant defense to the tissues at risk of brain infarction. Additionally, CINN had antioxidant effects by reducing the malondialdehyde levels in the ischemic brain tissues and the $\mathrm{Fe}^{3+}$ - induced lipid peroxidation in rat brain homogenate [26]. Thus, treatment with CINN could result in a marked reduction in postischemic accumulations of ROS and, consequently, attenuated the extent of lipid peroxidation, oxidative
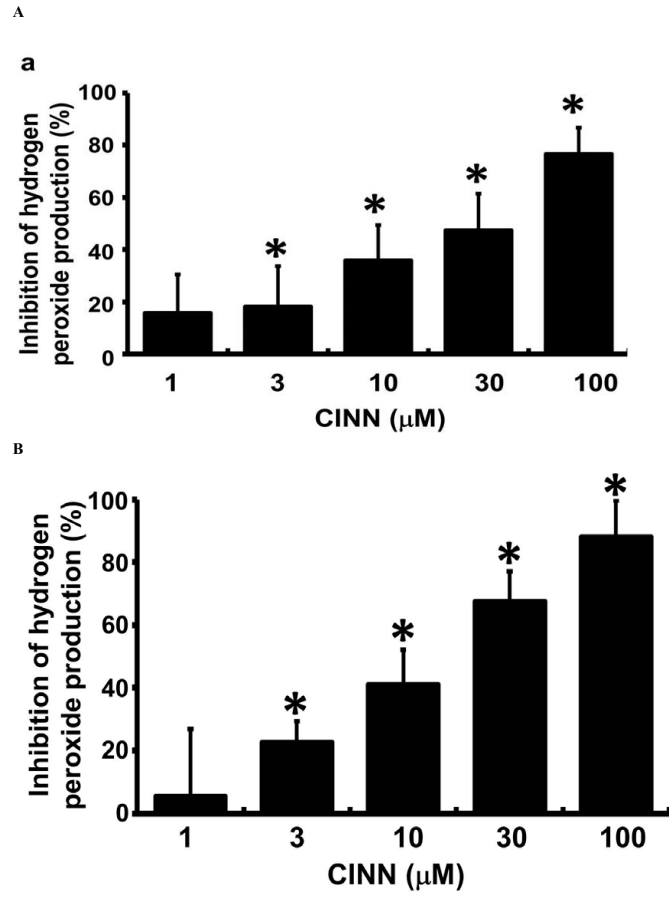

Figure 6: Inhibitory effects of cinnamophilin (CINN) on hydrogen peroxide production in PMA-treated neutrophils. (A) Cells were pretreated with CINN ( 1 to $100 \mu \mathrm{M}$ ) for 15 minutes, followed by staining with DHR-123. (B) Cells were cotreated with CINN and stimulated with PMA for 15 minutes, followed by staining with DHR-123. The hydrogen peroxide production of cell was determined by flow cytometric assay. Data are expressed by the mean \pm standard deviation (S. D.), and $n=6$ per group. ${ }^{*} P<0.05$ compared with vehicletreated controls by using the Kruskal-Wallis/Mann-Whitney $U$ tests. 
Citation: Lin YW, Tai SH, Tien CH, Huang SY, Chang CC, et al. (2013) Cinnamophilin Inhibits Neutrophilic Respiratory Burst and Protects Against Ischemia-Reperfusion Brain Damage. Pharmaceut Anal Acta 4: 272. doi: 10.4172/2153-2435.1000272

Page 5 of 6

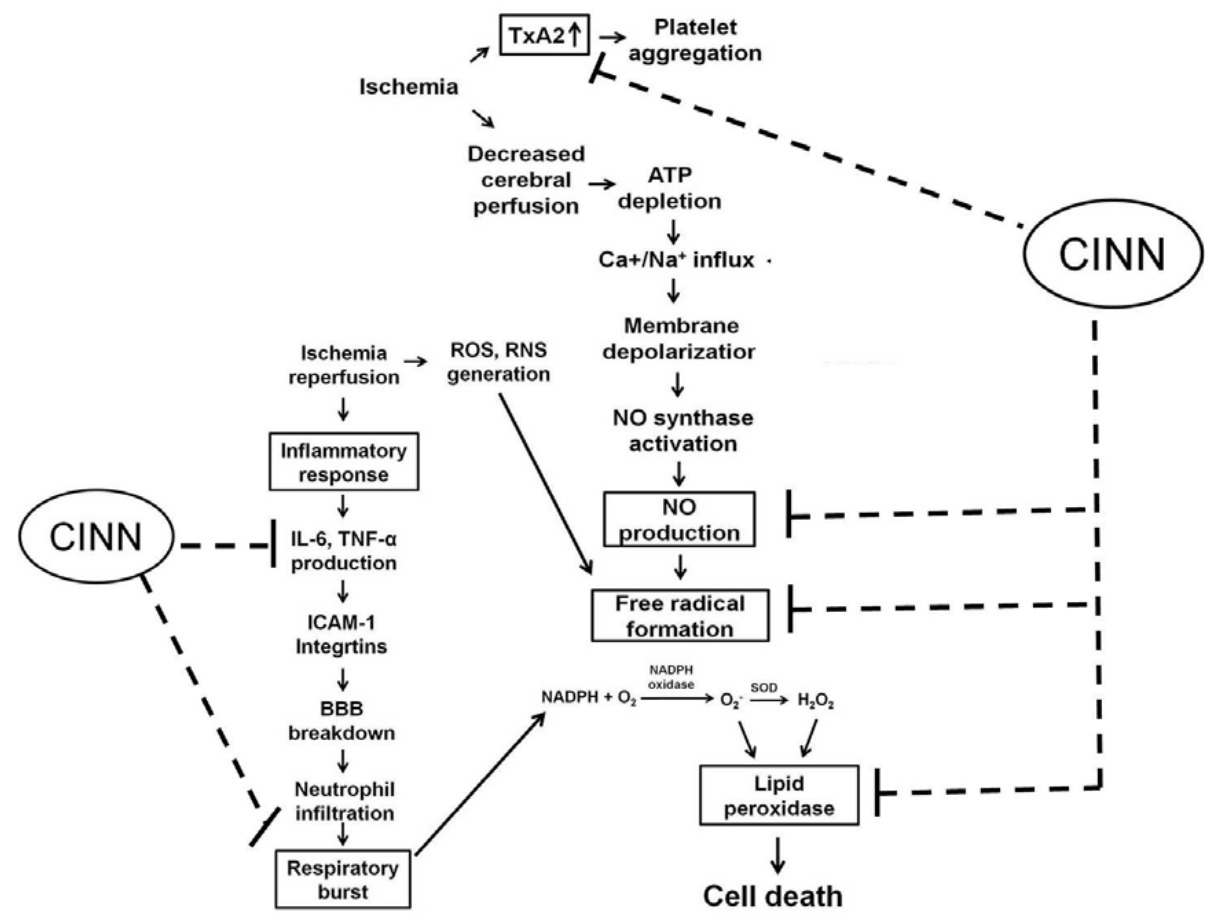

Figure 7: Cinnamophilin provides neuroprotection through pathways that involve inhibition of TXA2, antioxidant, anti-inflammatory effects and antirespiratory burst.

DNA damage, and the resultant neuronal deaths in the ischemic brain. The present study further demonstrated that CINN effectively reduced neuroinflammation by directly inhibiting neutrophilic respiratory burst in activated neutrophils.

The findings with no effect of CINN against MPO activity in the PMA-activated neutrophils are inconsistent with the in vivo data of CINN observed in a rat model of cerebral ischemia-reperfusion. This, however, indicates that molecular mechanisms of neuroprotective effect could be influenced by neuroprotective action in the model of whole animals. Thus, it was very possible that the CINN-mediated reduction in the MPO activity observed in vivo might have simply reflected its neuroprotection and anti-inflammatory actions against ischemic stroke. Other mechanisms, in addition to the inhibitory action against the neurotrophilic respiratory burst observed with CINN in vitro, might also have contributed to the CINN's ability against cerebral ischemiareperfusion insults observed in vivo. Thus, the in vivo and in vitro studies were essentially independent and might not establish a causal relationship. In addition, the dose-response levels in the present studies actually varied greatly between the in vivo and the in vitro studies.

Our results confirmed that administration of CINN significantly inhibited the PMA-induced $\mathrm{O}_{2}^{-}$. and $\mathrm{H}_{2} \mathrm{O}_{2}$ in neutrophils. However, CINN has no effect to inhibit the MPO activity in neutrophils. Thus, CINN did not directly attenuate the MPO pathway. In previous studies, calphostin C, an inhibitor of protein kinase C (PKC) can obviously reduce the He-Ne laser-induced respiratory burst of neutrophils [32], and the PMA is a stimulator for PKC-mediated NADPH oxidase and nitric oxide (NO) synthase during respiratory burst of neutrophils [33]. Therefore, it was very possible that CINN could also be a PKC inhibitor, and, thus, leading to a decrease in the $\mathrm{O}_{2}$ - production by reducing the $\mathrm{NADPH}$ oxidase activity, and this, however, needs further investigation.
It should be emphasized that fluorescent dyes such as DHR (for $\mathrm{H}_{2} \mathrm{O}_{2}$ ) and DHE (for superoxide radical) is not the best method of choice for radical detection, although it is more preferable in the cellular-based studies. The DHR and DHE actually react with many reactive oxygen species (and not only $\mathrm{H}_{2} \mathrm{O}_{2}$ or superoxide radical), and this non-specific integrity may leads to the production of other interfering substances which could also fluoresce and interfere. Superoxide reacts with DHE and produces a specific reaction product, the 2-hydroxy-ethidium which should be isolated to estimate accurately superoxide radical concentration. This could partly explain the decrease in superoxide radical with the significant high S. D. bar, as seen in Figure 2.

Our previous works have shown that CINN could be served as an effective free radical scavenger and antioxidant, and has multifaceted neuroprotective properties acting against cerebral ischemia-reperfusion injury. In stroke animals, CINN significantly reduced the infarction volumes, proinflammatory cytokine levels, nitric oxide production, and improved neurobehavioral outcomes as well (Figure 7). Therefore, CINN could prevent neuronal damage by its direct antioxidant and antiinflammatory effects after ischemia-reperfusion. In the present study, we further demonstrated that CINN inhibited neutrophilic respiratory bursts by directly attenuating the PMA-induced $\mathrm{O}_{2}-$. and $\mathrm{H}_{2} \mathrm{O}_{2}$, but not the MPO activity, and thus reduced post-stroke neutrophilic infiltration in the ischemic brain.

Further studies are needed to verify whether CINN would be a PKC inhibitor so as to reduce the NADPH oxidase activity and then to decrease the levels of $\mathrm{O}_{2}-$. More studies are also needed to demonstrate the neuroprotective mechanism of CINN in view of its potential role in the field of ischemic stroke.

\section{References}

1. Caplan LR, Hon FK (2004) Clinical diagnosis of patients with cerebrovascular disease. Prim Care 31: 95-109. 
Citation: Lin YW, Tai SH, Tien CH, Huang SY, Chang CC, et al. (2013) Cinnamophilin Inhibits Neutrophilic Respiratory Burst and Protects Against Ischemia-Reperfusion Brain Damage. Pharmaceut Anal Acta 4: 272. doi: 10.4172/2153-2435.1000272

2. Tissue plasminogen activator for acute ischemic stroke (1995). The National Institute of Neurological Disorders and Stroke rt-PA Stroke Study Group. N Eng J Med 333: 1581-1587.

3. Clark WM, Wissman S, Albers GW, Jhamandas JH, Madden KP, et al. (1999) Recombinant tissue-type plasminogen activator (Alteplase) for ischemic stroke 3 to 5 hours after symptom onset. The ATLANTIS Study: a randomized controlled trial. Alteplase Thrombolysis for Acute Noninterventional Therapy in Ischemic Stroke. JAMA 282: 2019-2026.

4. Furlan A, Higashida R, Wechsler L, Gent M, Rowley H, et al. (1999) Intra-arterial prourokinase for acute ischemic stroke. The PROACT II study: a randomized controlled trial. Prolyse in Acute Cerebral Thromboembolism. JAMA 282: 20032011.

5. Castellanos M, Leira R, Serena J, Pumar JM, Lizasoain I, et al. (2003) Plasma metalloproteinase- 9 concentration predicts hemorrhagic transformation in acute ischemic stroke. Stroke 34: 40-46.

6. Dijkhuizen RM, Asahi M, Wu O, Rosen BR, Lo EH (2002) Rapid breakdown of microvascular barriers and subsequent hemorrhagic transformation after delayed recombinant tissue plasminogen activator treatment in a rat embolic stroke model. Stroke 33: 2100-2104.

7. Montaner J, Molina CA, Monasterio J, Abilleira S, Arenillas JF, et al. (2003) Matrix metalloproteinase-9 pretreatment level predicts intracranial hemorrhagic complications after thrombolysis in human stroke. Circulation 107: 598-603.

8. Sumii T, Lo EH (2002) Involvement of matrix metalloproteinase in thrombolysisassociated hemorrhagic transformation after embolic focal ischemia in rats. Stroke 33: 831-836.

9. Wang YF, Tsirka SE, Strickland S, Stieg PE, Soriano SG, et al. (1998) Tissue plasminogen activator (tPA) increases neuronal damage after focal cerebral ischemia in wild-type and tPA-deficient mice. Nat Med 4: 228-231.

10. Babior BM (1978) Oxygen-dependent microbial killing by phagocytes (second of two parts). N Engl J Med 298: 721-725.

11. Badwey JA, Karnovsky ML (1980) Active oxygen species and the functions of phagocytic leukocytes. Annu Rev Biochem 49: 695-726.

12. Lipton $P$ (1999) Ischemic cell death in brain neurons. Physiol Rev 79: 1431 1568.

13. Babior BM, Kipnes RS, Curnutte JT (1973) Biological defense mechanisms The production by leukocytes of superoxide, a potential bactericidal agent. $J$ Clin Invest 52: 741-744.

14. Batot G, Martel C, Capdeville N, Wientjes F, Morel F (1995) Characterization of neutrophil NADPH oxidase activity reconstituted in a cell-free assay using specific monoclonal antibodies raised against cytochrome b558. Eur J Biochem 234: 208-215.

15. Park JW, Scott KE, Babior BM (1998) Activation of the leukocyte NADPH oxidase in a cell-free system: phosphorylation vs. amphiphiles. Exp Hemato 26: $37-44$.

16. Chopp M, Zhang RL, Chen H, Li Y, Jiang N, et al. (1994) Postischemic administration of an anti-Mac-1 antibody reduces ischemic cell damage after transient middle cerebral artery occlusion in rats. Stroke 25: 869-875.

17. Clark WM, Madden KP, Rothlein R, Zivin JA (1991) Reduction of centra nervous system ischemic injury by monoclonal antibody to intercellular adhesion molecule. J Neurosurg 75: 623-627.

18. Matsuo $Y$, Onodera H, Shiga $Y$, Nakamura M, Ninomiya M, et al. (1994) Correlation between myeloperoxidase-quantified neutrophil accumulation and ischemic brain injury in the rat. Effects of neutrophil depletion. Stroke 25: 14691475.

19. Matsuo Y, Onodera H, Shiga Y, Shozuhara H, Ninomiya M, et al. (1994) Role of cell adhesion molecules in brain injury after transient middle cerebral artery occlusion in the rat. Brain Res 656: 344-352.

20. Clemens JA (2000) Cerebral ischemia: gene activation, neuronal injury, and the protective role of antioxidants. Free Radic Biol Med 28: 1526-1531.
21. Kuroda S, Siesjö BK (1997) Reperfusion damage following focal ischemia: pathophysiology and therapeutic windows. Clin Neurosci 4: 199-212

22. Lee EJ, Lee MY, Chen HY, Hsu YS, Wu TS, et al. (2005) Melatonin attenuates gray and white matter damage in a mouse model of transient focal cerebral ischemia. J Pineal Res 38: 42-52.

23. Fabian $\mathrm{RH}$, Kent TA (1999) Superoxide anion production during reperfusion is reduced by an antineutrophil antibody after prolonged cerebral ischemia. Free Radic Biol Med 26: 355-361.

24. Yu SM, Ko FN, Wu TS, Lee JY, Teng CM (1994) Cinnamophilin, a nove thromboxane $\mathrm{A} 2$ receptor antagonist, isolated from Cinnamomum philippinense. Eur J Pharmacol 256: 85-91.

25. Yu SM, Wu TS, Teng CM (1994) Pharmacological characterization of cinnamophilin, a novel dual inhibitor of thromboxane synthase and thromboxane A2 receptor. Br J Pharmacol 111: 906-912.

26. Lee EJ, Chen HY, Hung YC, Chen TY, Lee MY, et al. (2009) Therapeutic window for cinnamophilin following oxygen-glucose deprivation and transient focal cerebral ischemia. Exp Neurol 217: 74-83.

27. Lee EJ, Chen HY, Lee MY, Chen TY, Hsu YS, et al. (2005) Cinnamophilin reduces oxidative damage and protects against transient focal cerebral ischemia in mice. Free Radic Biol Med 39: 495-510.

28. Hsiao G, Teng CM, Sheu JR, Cheng YW, Lam KK, et al. (2001) Cinnamophilin as a novel antiperoxidative cytoprotectant and free radical scavenger. Biochim Biophys Acta 1525: 77-88.

29. Chen TY, Tai SH, Lee EJ, Huang CC, Lee AC, et al. (2011) Cinnamophilin offers prolonged neuroprotection against gray and white matter damage and improves functional and electrophysiological outcomes after transient focal cerebral ischemia. Crit Care Med 39: 1130-1137.

30. Lee EJ, Wu TS, Lee MY, Chen TY, Tsai YY, et al. (2004) Delayed treatment with melatonin enhances electrophysiological recovery following transient foca cerebral ischemia in rats. J Pineal Res 36: 33-42.

31. Clark WM, Rinker LG, Lessov NS, Hazel K, Hill JK, et al. (2000) Lack of interleukin- 6 expression is not protective against focal central nervous system ischemia. Stroke 31: 1715-1720.

32. Duan R, Liu TC, Li Y, Guo H, Yao LB (2001) Signal transduction pathways involved in low intensity $\mathrm{He}-\mathrm{Ne}$ laser-induced respiratory burst in bovine neutrophils: a potential mechanism of low intensity laser biostimulation. Lasers Surg Med 29: 174-178

33. Vouras C, Dailianis S (2012) Evidence for phosphatidylinositol-3-OH-kinase (PI3-kinase) involvement in Cd-mediated oxidative effects on hemocytes of mussels. Comp Biochem Physiol C Toxicol Pharmacol 155: 587-593. 